
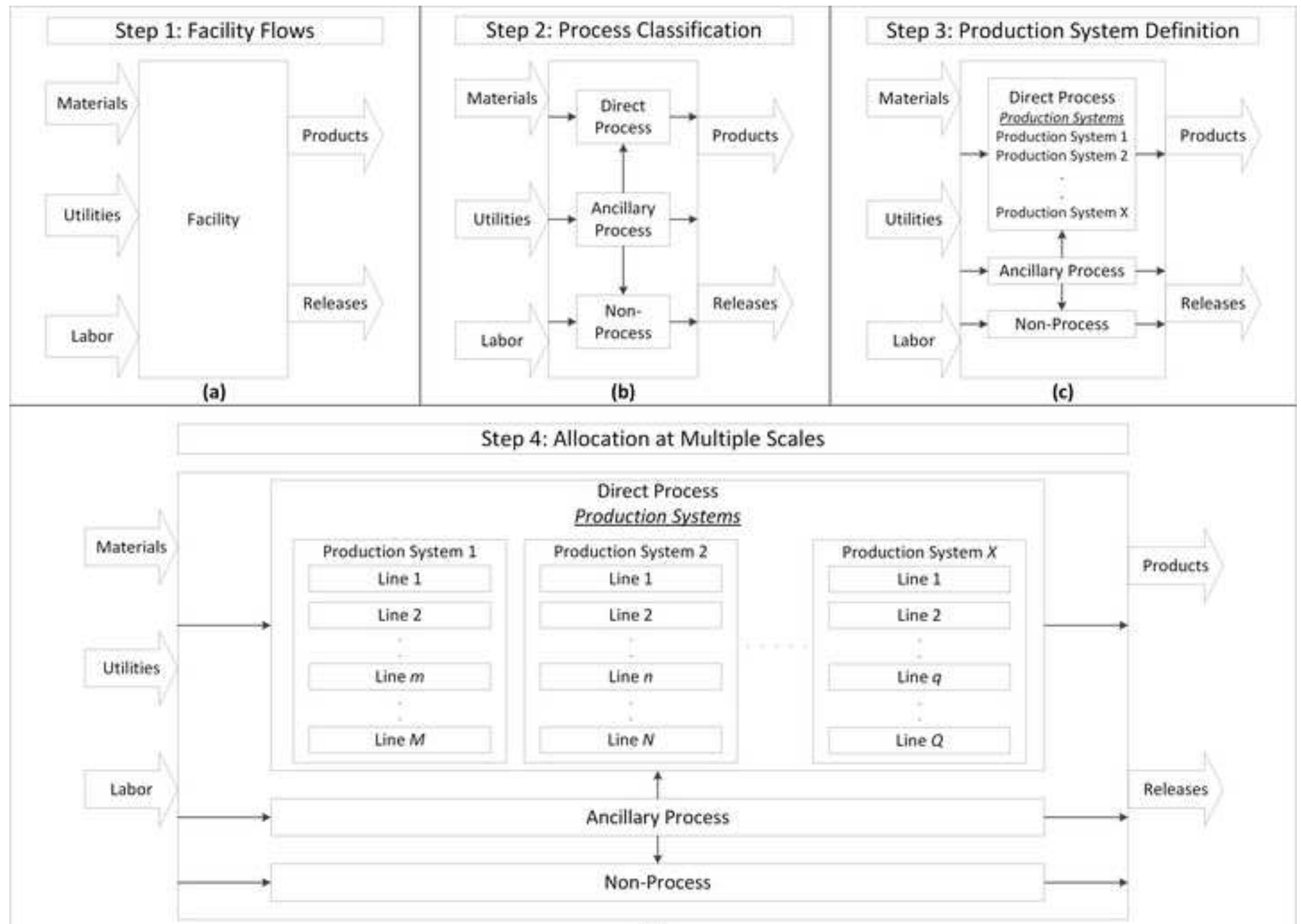

(d) 


\title{
Industrial Process System Assessment: Bridging Process Engineering and Life Cycle Assessment through Multiscale Modeling
}

\author{
Debalina Sengupta ${ }^{a}$, John P. Abraham ${ }^{b}$, Manuel Ceja ${ }^{c}$, Michael A Gonzalez ${ }^{b}$, Wesley W. \\ Ingwersen $^{b^{*}}$, Gerardo J. Ruiz-Mercado ${ }^{\text {b }}$, Raymond L. Smith ${ }^{\mathrm{b}}$ \\ a Artie McFerrin Department of Chemical Engineering, Texas A \& M University, College \\ Station, TX \\ ${ }^{\mathrm{b}}$ United States Environmental Protection Agency, National Risk Management Research \\ Laboratory, Sustainable Technology Division, Cincinnati, $\mathrm{OH}$ \\ ${ }^{\mathrm{c}}$ Procter \& Gamble, Family Care, Cincinnati, $\mathrm{OH}$ \\ * Corresponding Author
}

\begin{abstract}
Conducting life cycle assessments (LCA) of complex multi-line, multi-product manufacturing facilities can be challenging. There is no standard method to assign or allocate resource use and environmental impacts to the individual products. The Industrial Process System Assessment method is presented to address this gap in current approaches for LCA allocation by making use of a multiscale model of process systems. Algorithms for the allocation procedure rectify process, ancillary, and non-process data from a facility such that inputs (e.g., raw materials) and outputs (e.g., emissions) can be assigned to a particular product from a particular line. The allocation approach demonstrates systematic multiscale process model development to avoid arbitrary allocation assumptions. This standardized method will enable LCA practitioners and process engineers to more clearly differentiate resource use and environmental impacts of co-products from complex facilities. The model could be further extended to provide product allocation based on individual unit operations when data are available.
\end{abstract}

Keywords: Industrial Process Systems Assessment, IPSA, Allocation, Life Cycle Assessment, Life Cycle Inventory Analysis, LCA, LCI, Process Engineering, Multiscale Modeling

\section{Introduction}

The value of performing a Life Cycle Assessment (LCA) of a product depends on the extent to which it increases the ability to understand the environmental consequences from the technological systems responsible for its production as well as the up- and down-stream 

and energy flows and environmental releases associated with a product along its entire value chain, known as a Life Cycle Inventory (LCI), are quantified to enable LCA and support effective decision making (Jiménez-González et al., 2004; Jiménez-González et al., 2000; Lindfors, 1995).

When facilities manufacture multiple products (or have multiple lines manufacturing the same product), developing an accurate LCI for just one of those products (or one of those lines) can be difficult. In such cases, due to unavailability of detailed information, flow and release data are often aggregated across the multiple products (or lines) at a facility level and assigned to the particular product (or line) of interest. The procedure of assigning/estimating each individual product's share of the responsibility for an LCI is called coproduct allocation (Löfgren et al., 2011; USEPA, 1993).

LCA practitioners have proposed various coproduct allocation techniques (GonzálezGarcía et al., 2013); however, there is no standardized method and the primary recommendation is to avoid allocation if at all possible (ISO, 2006; USEPA, 2006) by developing greater process details. According to the ISO 14044 standards, "dividing the unit process to be allocated into two or more sub-processes and collecting the input and output data related to these sub-processes" is a means to avoid allocation (ISO, 2006).

The Industrial Process System Assessment (IPSA) method provides an approach which avoids allocation through process subdivision where possible, and allocates other inputs and releases where necessary. To outline the remaining parts of this contribution, the next section will describe background information on allocation methods in LCA, Process System Engineering approaches, and how IPSA bridges the two. A section containing a detailed step by step description of the IPSA method follows, with a final section suggesting additional uses and extensions of the method.

\section{Background}

Creating an LCI and assigning input and release flows to a product can pose challenging issues in multi-product or multi-production line systems. From a discussion by Ekvall and Finnveden (2001) on the allocation methods described in ISO 14041, subdivision (i.e., avoiding allocation), system expansion, and allocation based on physical causal relationships were identified as the primary methods for properly allocating inputs and outputs to a product. However, system expansion is not universally recommended because it involves using potentially hypothetical flows from processes outside of the supply chain being studied. Therefore, Heijungs and Guinee (2007) argue for always partitioning resource use and release flows when allocation is necessary. The various suggestions illustrate that a standard method for applying allocation for complex industrial systems has not been developed. Without a defined 

is limited.

A generalized method for the allocation of energy use and emissions in petroleum refineries with multiple products was proposed by Wang et al. (2004). In this reference, the refinery is divided into subprocesses where each sublevel represents an individual refining process; however, energy use and emissions had to be allocated in some cases. The results from this study show that different values for energy use and emissions are obtained when the allocation to products is performed by mass-, energy content-, and market value-based methods. When facility level allocation and subprocess level allocation results are compared, substantially different values are obtained.

Recently, the UNEP-SETAC Life Cycle Initiative brought together experts to define principles for life cycle data collection and management, which are captured in the Shonan Guidance principles (Sonnemann and Vigon, 2011). According to the Shonan principles, when more than one unit process is combined into a single process model, this is known as vertical aggregation. Vertical aggregation is often performed to protect confidential business information. The specific case of vertical aggregation that is based on a series of unit operations within a single facility is further defined as technical aggregation. Technical aggregation is complicated in the case of complex facilities where there are multiple products being manufactured because there are often multifunctional unit operations (the same unit operation is shared between products). When engineering knowledge is limited, or data are unavailable for modeling the precise contributions of multifunctional processes to specific products, assumptions must be made and subsequent decisions made to allocate the inputs and outputs of these processes to the products.

The Shonan Guidance principles do not include a standardized methodology of allocation for technically aggregated, multifunctional processes. In the LCA literature, allocation issues have been thoroughly explored, but the focus has been on identifying appropriate causality for allocation; in other words, whether allocation should be done based on different physical (e.g., mass or energy) or economic characteristics of the co-products (Azapagic and Clift, 1999a, b, 2000; Curran, 2007; Ekvall and Finnveden, 2001). However, even after arriving at an appropriate cause for allocation, there are still a large number of modeling choices to be made to allocate burdens to products in complex manufacturing systems. Despite the ubiquity of these types of production systems in modern manufacturing, a standardized method for allocation applicable to complex manufacturing facilities has not been proposed. Allocation strategies are implemented for reasons other than developing inventory for product life cycle assessment. Additional uses of allocation include process quality control and fault assessment (Jin et al., 2010). In such systems, linear state space models (mass-based) are employed to allocate quality information datacollection points, account for facility internal structures, and to detect out of control conditions. 
Allocation methods used in the manufacturing and chemical industries have been implemented for process control, optimization, product quality, energy use, and the distribution of feedstock needs, utilities consumption, and releases to products (Jin et al., 2010; Sammons Jr et al., 2008; Wang et al., 2004). Allocation methods for products can be based primarily on mass, energy content, or market value of particular products from a facility. Allocation strategies can be applied at the facility level where it is assumed that equal or proportional feedstock, energy, and emissions (direct and indirect) are attributed during manufacturing to all facility products. Alternately, facilities can be divided into subprocesses for each product and these subprocesses can be modeled to avoid allocation. The subprocess level modeling helps to reveal some additional burdens (e.g., energy and emissions) associated with certain facility products that are otherwise overlooked in facility-level allocation.

Some challenges exist regarding data availability and accessibility at the sub-facility and sub-process levels. When possible, assigning energy and material use should be done at the lowest sub-process level. If detailed process data are not available, there are some methods such as linear programming (LP) models and discrete event simulation to approximate or convert facility level data to subprocess level data (Löfgren and Tillman, 2011; Wang et al., 2004). These approaches (e.g., linear assumptions, black box models) can be calibrated to an actual facility or an aggregate of facilities.

Traditionally, decisions for manufacturing processes are made based on product needs or process improvements, e.g., installation of more efficient equipment, cost minimization, etc. Process Systems Engineering (PSE) enables the understanding and development of systematic procedures for the design and operation of process systems, ranging from microsystems to industrial scale continuous and batch processes (Cameron et al., 2008; Grossmann and Westerberg, 2000). Figure 1 shows the multiple scales of process engineering required in the synthesis of a product. In addition to the spatial and temporal scales shown, for example for Unit Operations, a finer detailed scale (in this case Single and Multi-phase Systems) can also be considered (Cameron et al., 2008). The finer scale can be used for a granular evaluation of the system above it, for instance, in this example in terms of its number and type of balance volumes, degree of aggregation, mechanisms related to flows, reaction, heat, mass and momentum transfers, etc. The idea of larger and finer scales as depicted in Figure 1 applies to various types of processes and products.

In the consumer products sector, core processes such as the manufacture and packaging of final products are under the immediate control of the producer. These processes often play a major role in affecting both upstream and downstream environmental impacts, because their material and energy requirements affect upstream and downstream facilities. The characteristics of the final product and its packaging affect its performance in the use phase and the ultimate end-of-life impacts. In recent years, LCA has been used as a screening level tool for development of sustainable supply chain tools (Power, 2009). LCA has been identified as one of 
the key tools for sustainable engineering in the future (Burgess and Brennan, 2001; Grossmann, 2004; Wan Alwi et al., 2014). However, practitioners often face their greatest difficulty in the creation of a robust inventory of all the necessary raw material extraction, manufacturing, distribution, use, waste treatment, and disposal processes to model a complete product life cycle. This is due to the expansive scope and yet extensive level of detail required to model the "cradleto-grave" environmental impacts, or other economic and social impacts associated with a product. LCA studies compiled with robust and reliable inventory data using final product manufacturing inputs can reveal improvement opportunities for the sustainable design of consumer products which can be addressed by the manufacturer. Compared to traditional LCA, the multiscale process system model defined by IPSA provides a unique and novel approach which allows for a detailed subdivision of the process system where multi-product systems can be isolated and the inputs and outputs attributed to the product of interest. As shown in the following section, the IPSA method provides a structured method for assessing the inputs and outputs related to a product of interest when the intention is to compare products from specific production lines in one or more complex industrial facilities. 


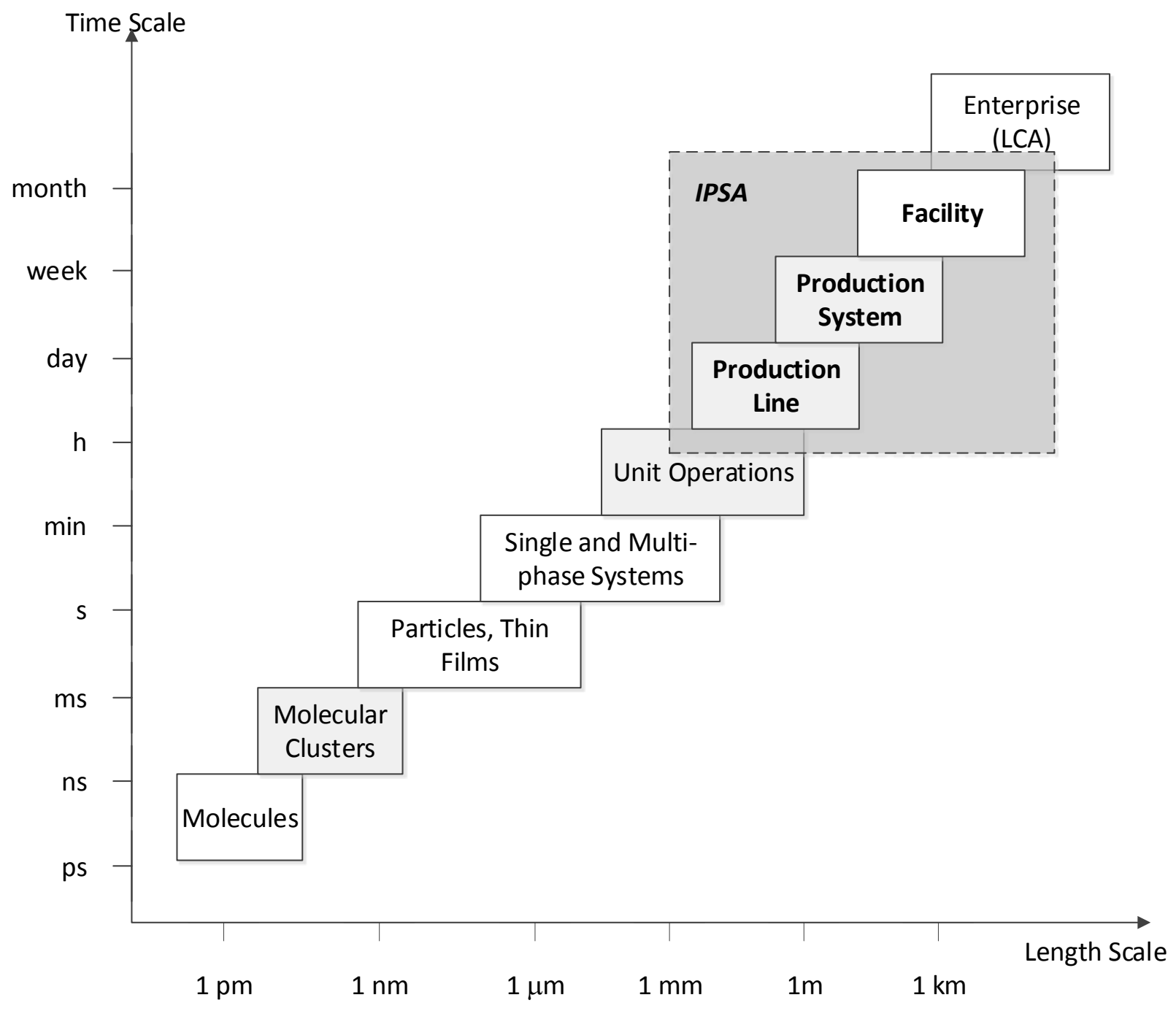

Figure 1. Multiple scales of process engineering (adapted from Cameron et al., 2008); shaded area includes the scales modeled in the current IPSA method.

\section{Methods}

To understand a real system and complete this research, data were gathered from two operating paper product facilities in North America run by The Procter \& Gamble Company. The facilities selected differ in geographical location, process technology, and incorporate multiple production lines. After development and validation of this method relative to the chosen production lines, the next step is to make this method generic so a similar analysis approach can be used for evaluating the sustainability of products from any industrial (manufacturing) process system.

The IPSA method, consisting of four steps, was developed for the purpose of obtaining inputs and outputs related to a product at desired levels of granularity for a process system. This is done in the context of a LCA, which contains three distinct sections, the cradle-to-gate (raw 
materials to plant boundary), gate-to-gate (raw materials conversion within the plant boundary to product), and gate-to-grave (product use, and final disposal) (Dominguez-Ramos et al., 2014). The need for the IPSA method directly arose from the need to supply detailed information related to the production of paper towels at the $P \& G$ facilities to complete the gate-to-gate portion of the LCA. An additional need for the development of the IPSA method arose from the requirement to supply flow rate information related to the product of interest to new integrated metrics being developed; Green Net Value Added (GNVA) and Fisher Information (FI) (Ingwersen et al., 2014; Weisbrod et al., 2013). Once the IPSA method was defined and a basic tool developed, it was validated with production related data from P\&G's Box Elder, UT and Albany, GA paper product manufacturing facilities. A rigorous data acquisition process involving a core team of engineers, scientists, and other plant resources was utilized for the model validation. Lessons learned from the validation process were used to refine the IPSA method and facility models, while additional ideas for future automation and expansion of the model were captured.

The IPSA method provides a rigorous modeling and data collection method to bridge the gap that exists in traditional LCA allocation methods. This is accomplished by aligning a multiscale model of a process system with the steps in assigning inputs and outputs to the product from the system. Process systems knowledge has been used to divide a process from facility level to sub-process level and to obtain information at multiple scales. In addition, the sub-processes within a facility not directly associated with the manufacturing of the product, but providing essential support to the production process, are also considered in the multiscale model formulation. Then, a production line combination that is responsible for manufacturing a particular product within the multi-product production system was identified for model application.

Development of a multiscale model for industrial process systems makes use of the following steps used for conventional non-multiscale process systems: 1) model goals and definition, 2) model conceptualization, 3) modeling data needs and sources, 4) model building and analysis, 5) model verification, 6) model solution, and 7) model calibration and validation (Cameron et al., 2008). A multiscale model is composed of partial models or submodels. In Figure 1, the production line, production system, and the facility are submodels of the multiscale model of a process system. A well-posed modeling problem for each submodel is required where it can be classified into a mechanistic or empirical, stochastic or deterministic, lumped or distributed parameter, linear or non-linear, continuous or discrete, or hybrid model. In most cases, when mechanisms or underlying phenomena of a process are not available for creating a fully determined (i.e., "white" box) model, an input-output "black" box, or a hybrid "grey" box model is created (Cameron et al., 2008). This is done primarily because only aggregate data from the overall process system are available, and not necessarily from subsystems in the corresponding partial models. The development of the multiscale model for the P\&G paper product manufacturing process gives rise to partial models as described in the four steps below. 


\subsection{Multiscale Model Development for Industrial Processes}

Many production facilities perform multiple operations, some of which are directly related to the manufacturing of the product(s) and others which provide support for the facility. To develop a method for relating the inputs and outputs of a facility to its products, it is necessary to draw a distinction between the various operations within the facility.

Often, a direct link is not sought or available for connecting input and output flows of a facility to its products. For some inputs and outputs, an overall facility tracking mechanism may be in place. Examples of such input flows include electricity, water, or natural gas use at a facility tracked with monthly or quarterly bills. Output examples include the volume of water effluent released to a municipal wastewater treatment facility. These inputs and outputs are attributed using a top-down approach. In other cases, the inputs and outputs may be tracked or estimated at the equipment level, and aggregated to compute the quantities for the overall facility, e.g., the materials used to make a product in a particular machine, or the environmental releases computed from the emission parameters of a boiler. These inputs and outputs are allocated using a bottom-up approach.

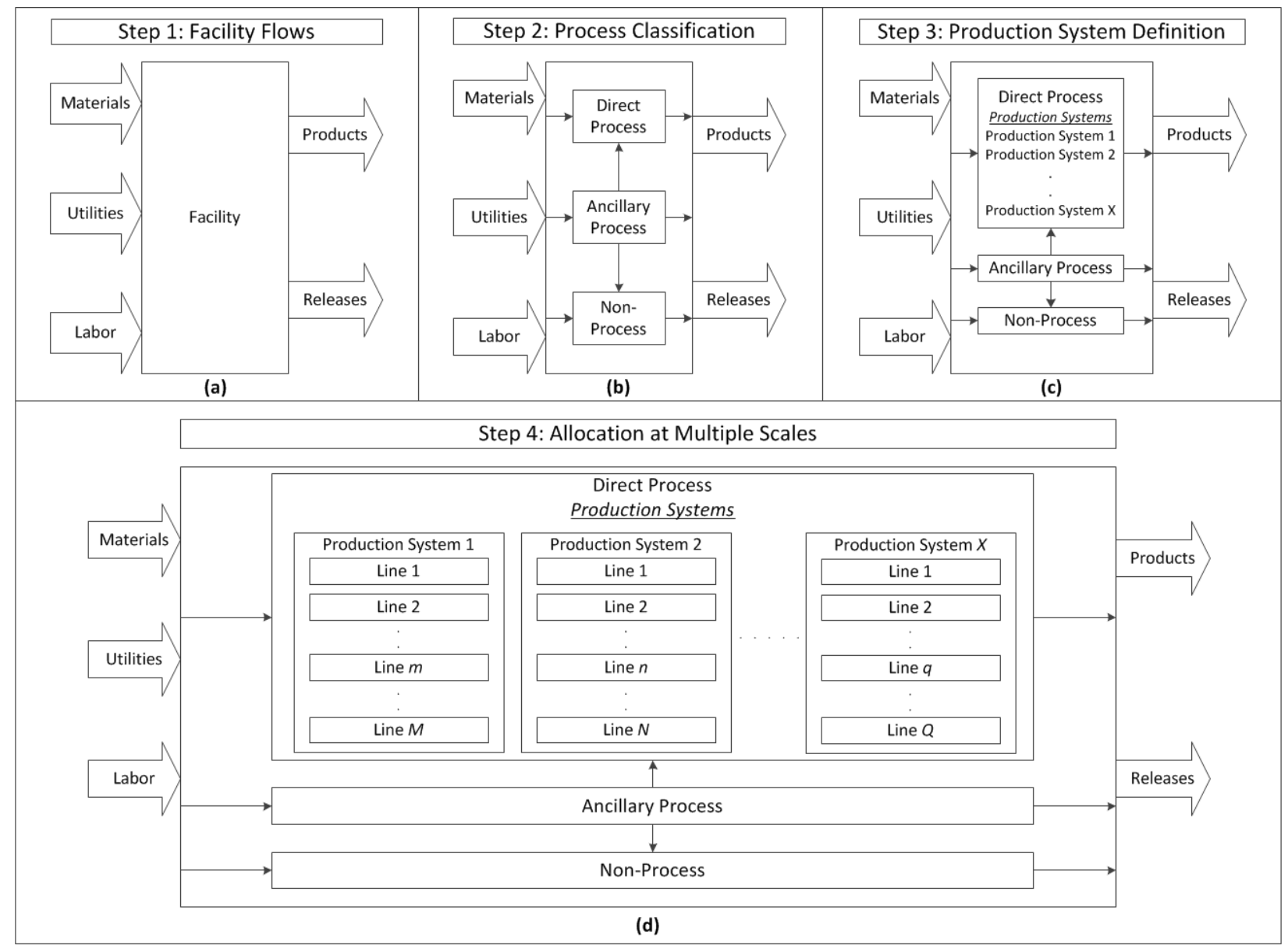


Figure 2 (a) Step 1: Facility Flows, (b) Step 2: Process Classification, (c) Step 3: Production System Definition, and (d) Step 4: Allocation at Multiple Scales.

IPSA utilizes the multiscale process design model in Figure 1 as a guide for subdivision of the procedure into four steps. These steps help define the submodels within the overall "facility level" information and relate it to "production line" level information or vice versa. The current iteration of the IPSA method is limited to the "production line" scale because it is the level of granularity desired for the sustainable supply chains methods and tools being developed by P\&G and US EPA. The same approach can be extended to finer levels of granularity, e.g., comparing two unit operations such as similar process transformation equipment within two different production lines.

The various steps in the IPSA model development are shown in Figure 2. Table 1 shows the corresponding parameters particular to the P\&G case study. Step 1 is shown in Figure 2(a), which presents Facility Flows. At the Facility Flow level of the multiscale model a listing and quantification of all inputs and outputs of the facility are reported. The "Materials" flow represents all the inputs that are purchased for the facility, and contribute in the physical constitution of the final product. The "Utilities" are the flows associated with purchased or onsite generation of materials and energy for supporting the process system within the facility. The "Labor" flow provides the human labor and contracted service needs of the facility that enable the functioning of the process system. In conjunction with traditional engineering accounting, the input of Labor needs to be separated into direct labor (required for the running of direct or ancillary process equipment) and indirect labor (required for other non-process activities) that are required within the facility boundary. The Labor flow includes contracted or purchased services which provide essential service for the manufacturing process, such as waste disposal contracts, cafeteria services, etc. As such, IPSA is able to fulfill the needs of both the LCA and integrated metrics computation by including the capability of these three input categories in the model. A comprehensive and detailed list of inputs and outputs is desired at Step 1; this provides the ability to determine the upstream and downstream life cycle impacts of the inputs and the outputs. For example in the paper towel case study, in addition to the total quantity of pulp, the types of pulp and the sourcing information for each is desired to be able to account for and assess the supply chain attributes upstream of actual towel manufacturing. Similarly, for the total electricity purchased by the facility, it is desirable to know and make use of the fuel based composition (primary energy source) of the electricity supplier for estimating resource use and the emissions from its production. The direct labor hours utilized in the facility is important for calculation of the labor costs while computing the green net value associated with the product of interest.

Figure 2(b) depicts Step 2 - Process Classification of the model. In this step, the operations of a facility are classified as "Direct Process", "Ancillary Process" and "Non-Process" based on their function. The Direct Processes refer to the physical operations within a facility 

equipment which consume resources (inputs) and produce products and releases during manufacturing. This category is typically initiated with receipt and storage of raw materials, continues through preparation of a raw material, e.g., wood pulp mixing with water and additives for the paper towel manufacturing process, and progresses through various manufacturing steps resulting in a final, packaged product ready for shipment from the facility warehouse, e.g., a pallet of paper towels loaded onto a tractor trailer. The Non-Process category of operations is frequently considered as indirect or overhead functions at a facility. Typical Non-Process operations can include: purchasing, human resources, payroll, cafeteria food services, health and safety, management, etc. Although labor is the major input to Non-Process operations, these operations are also responsible for consuming facility inputs (e.g., electricity, water, etc.), as well as responsible for a portion of the facility's releases. The Ancillary Processes are comprised of the unit operations which provide support to the Direct Processes and Non-Processes. In this category of operations, the inputs to an ancillary process are converted and the transformed product is shared among the Direct Processes and Non-Processes within the facility. For example, a facility's boiler transforms natural gas or coal to steam, which is critical to paper manufacturing and at the same time furnishes steam to the heating, ventilation, and air conditioning (HVAC) system which heats the office spaces, or supplies steam to heat water for the cafeteria, etc. On-site water treatment may treat discharges from pulp dewatering equipment (a direct process step) as well as that from cafeteria operations. In general, 100 percent of every input to, and release from, shared operations will need to be proportioned between the Direct and Non-Process categories. In effect, this results in the creation of two, separate and distinct life cycle inventories (LCIs). If one combines the two LCIs, the result is a "traditional" or conventional LCI at the facility level. Thus, identification of the Direct Processes, Ancillary Processes, and Non-processes is a crucial step to divide the facility into its three main functional areas.

Table 1. Parameters in the Paper Towel Case Study for P\&G

\begin{tabular}{|c|c|c|}
\hline Location & IPSA Step & Parameter \\
\hline \multirow[t]{4}{*}{ Albany, GA } & Step 1 & $\begin{array}{l}\text { Inputs: Pulp, Chemicals, Electricity, Natural Gas, Biomass, Oils, } \\
\text { Water } \\
\text { Outputs: Paper Towel, Paper Tissue, Releases }\end{array}$ \\
\hline & Step 2 & $\begin{array}{l}\text { Direct Process: Paper Production Processes } \\
\text { Ancillary Processes: Boiler System, Water Treatment System, } \\
\text { Wastewater Treatment System } \\
\text { Non-Process: Cafeteria, Office Space, etc. }\end{array}$ \\
\hline & Step 3 & $\begin{array}{l}\text { Production System: } X=2 \\
\text { Make Production System } \\
\text { Pack Production System }\end{array}$ \\
\hline & Step $4^{*}$ & $\begin{array}{l}\text { Make Production Lines: } M_{1} \\
\text { Pack Production Lines: } N_{l}\end{array}$ \\
\hline Box Elder, UT & Step 1 & Inputs: Pulp, Chemicals, Electricity, Natural Gas, Water \\
\hline
\end{tabular}


Outputs: Paper Towel, Paper Tissue, Releases

Step 2 Direct Process: Paper Production Processes

Ancillary Processes: Boiler System, Water Treatment System, Wastewater Treatment System

Non-Process: Cafeteria, Office Space, etc.

Step $3 \quad$ Production System: $X=2$

Make Production System

Pack Production System

Step $4 * \quad$ Make Production Lines: $M_{2}$

Pack Production Lines: $N_{2}$

The actual values of $M_{1}, N_{1}$ and $M_{2}, N_{2}$ are not disclosed to protect proprietary information.

Step 3 - Production System Definition of the model is illustrated in Figure 2(c). Several production systems combine to comprise the Direct Process. Production systems are connected in series and/or in parallel to obtain the product of interest. Step 3 identifies the production systems in greater detail. Facility inputs are distributed among these production systems, and facility outputs are a combination of the outputs from the production systems. This scheme allows for specifying that production systems can manufacture an intermediate product, which can be accumulated in an intermediate storage area. Using this approach intermediate output(s) from one production system will be utilized by a subsequent production system, until the final product output is obtained. Figure 2(c) shows a general case where there are $X$ number of production systems. For the $P \& G$ production facilities, there are two production systems classified as Direct Processes, the "Make Production System" and the "Pack Production System". The Make Production System uses pulp, chemicals, and utility inputs to produce intermediate products defined as parent rolls for use in tissue and towel products. Parent rolls can then be used immediately or stored for later use on a demand basis by the Pack Production System for the final tissue and towel production, including packaging.

Figure 2(d) indicates Step 4 - Allocation at Multiple Scales of the model development. This step further divides each production system into production lines. A serial-parallel combination of unit operations constitutes a production line. Multiple production lines are capable of producing similar intermediate or final products. For example, the Make Production Lines are capable of producing parent rolls, which are subsequently converted by the Pack Production Lines into final product. Each individual line is allocated resources, which are dependent on the unit operations and the demand of the intermediate or final product of interest from the line. The figure shows "Production System 1" with $M$ production lines, "Production System 2" with N production lines, and "Production System $X$ " with $Q$ production lines.

The IPSA method includes evaluation of existing facility information related to different inputs and outputs, which is referred to as Step 0 - Recursive Attribute Allocation and is described in the sections below. Step 0 allocation describes how the production systems, energy, and fuel tracking within the different model scales, emissions, water use and disposal, and solids 

account for losses or unaccounted material and energy flows at each step of the multiscale model, but that functionality is not yet fully developed. The loss accounting method provides useful information for estimating process efficiencies and will be integrated into the IPSA method in the future.

After developing the facility multiscale model as defined by completing Steps 1 through 4 , the inputs and outputs to the facility are distributed to the production lines, which is the finest granularity in each production system studied. However, to obtain the relationship between two production lines from subsequent production systems, scaling of inputs or outputs is required. This relationship is computed from a Step 0 calculation. In Figure 3, the scaling is shown with respect to the $\mathrm{P} \& \mathrm{G}$ paper towel case study. For a given production schedule, the Make Line $m$ produces $I_{m}$ intermediate parent rolls, and the Pack Line $n$ processes $I_{n}$ intermediate parent rolls. $I_{m}$ and $I_{n}$ are both measured quantities expressed as mass per unit time for the respective production lines. The intermediate storage holds the parent rolls which are further converted to the main product at a later time. For determining the reference flow of product from a combination of these two production lines within the same production system, two scenarios are considered as shown in Table 2.

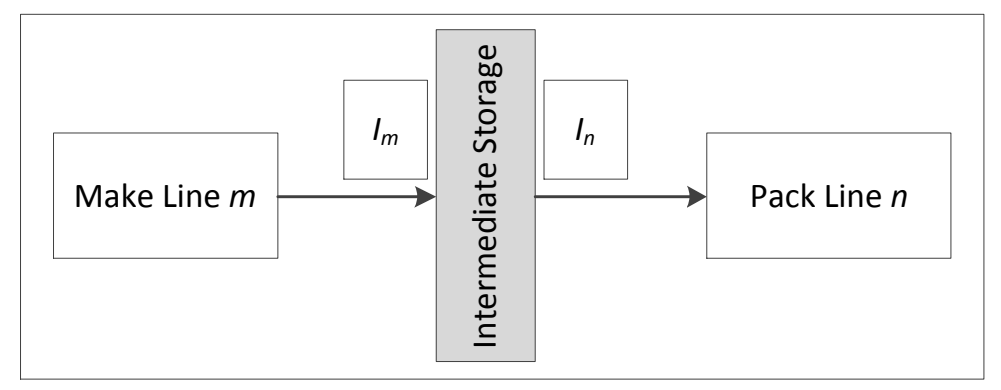

Figure 3. Scaling method for computing the intermediate ratio between the "Make Line $m$ " and the "Pack Line $n$ ".

Scenario 1: $I_{m} / I_{n}<1$, then $I_{m}$ is the limiting factor. In this case, it is assumed that the Pack Line $n$ uses all the $I_{m}$ intermediate and the remaining $I_{n}-I_{m}$ rolls are supplied by other Make Lines in the Make Production System. Thus, the fraction of intermediates converted by the Pack Line $n$ is $I_{m} / I_{n}$. This fraction is used to scale the inputs and outputs from the Pack Line in consideration.

Scenario 2: $I_{m} / I_{n}>1$, then $I_{n}$ is the limiting factor. In this case, it is assumed that all the intermediate converted in Pack Line $n$ is produced in the Make Line $m$. The intermediates $I_{m}-I_{n}$ not used in the Pack Line $n$ will be used by other production lines in the Pack Production System. Thus, the fraction of intermediates converted by the Pack Line $n$ is $I_{n} / I_{m}$. This fraction is used to scale the inputs and outputs from the Make Line in consideration. 
Table 2. Multiplication factors for scaling of intermediates and products from a combination of Make Line $m$ and Pack Line $n$

\begin{tabular}{ccc}
\hline Scenario & Multiplication Factor & Multiplied to Inputs and Outputs of \\
\hline Scenario 1: $I_{m} / I_{n}<1$ & $I_{m} / I_{n}$ & Pack Line $n$ \\
Scenario 2: $I_{m} / I_{n}>1$ & $I_{n} / I_{m}$ & Make Line $m$ \\
\hline
\end{tabular}

The scenarios above consider only two production systems as in the $P \& G$ paper towel case study (depicted in Figure 4), but the method can be applied to more complex facilities with an $X$ number of production systems. Either a top-down or a bottom-up approach is needed to obtain the data for the multiscale model development of Steps 1-4. Thus, depending on the type of input or output, either Step 1 or Step 4 may be used as a starting point for the model development.

\subsection{Mathematical Formulation of Multiscale Model}

The purpose of the IPSA method is to generate the inputs and outputs per unit of a product obtained from production facilities consisting of $m$ Make Lines and $n$ Pack Lines as defined by the four step multiscale model development described above. Figure 4 shows a schematic of the subdivision of inputs in this $P \& G$ case study. Step 1-4 allocations correspond to Steps 1-4 of the multiscale model. $i_{m, n}$ is the input, which describes the production from the combination of a particular Make Line $m$ and Pack Line $n$ combination. For the two Production Systems in the P\&G case study, a total of six terms (as shown along the bottom of Figure 4) were computed for obtaining the value of $i_{m, n}$. For an industrial system with $X$ Production Systems, the total number of terms in $i_{m, n}$ is $2 X+2$. An explanation of the different allocation steps is provided below with respect to the inputs to the process. A similar set of equations will apply for the outputs. To prevent a redundant presentation of information a detailed decomposition of the system outputs has been omitted in this manuscript. The final equations relating the overall facility output to $o_{m, n}$ are provided in a later section.

\subsubsection{Step 1 Allocation:}

In the Step 1 allocation, the inputs to the facility are determined and listed. Each input is denoted with $i$ and measured in consistent units of flow per unit time depending on the type of input, such as mass, volume, or energy. The IPSA method is applicable to various time periods, but a period of one year (either calendar or fiscal) is suggested for measuring inputs to a facility; data on this periodicity is generally readily available from facility datasets. If it is not available, monthly, weekly, or daily data may need to be aggregated to arrive at an annual value for mass, energy, or volume. This aggregation will normalize for seasonal or operational variations. 


\subsubsection{Step 2 Allocation:}

In the Step 2 allocation, the inputs are distributed to the Direct Process (DP), Ancillary Process (AP), and Non-Process (NP).

Distribution of Facility Inputs to Direct Process, Ancillary Process, and Non-Process

For any given input, $i$,

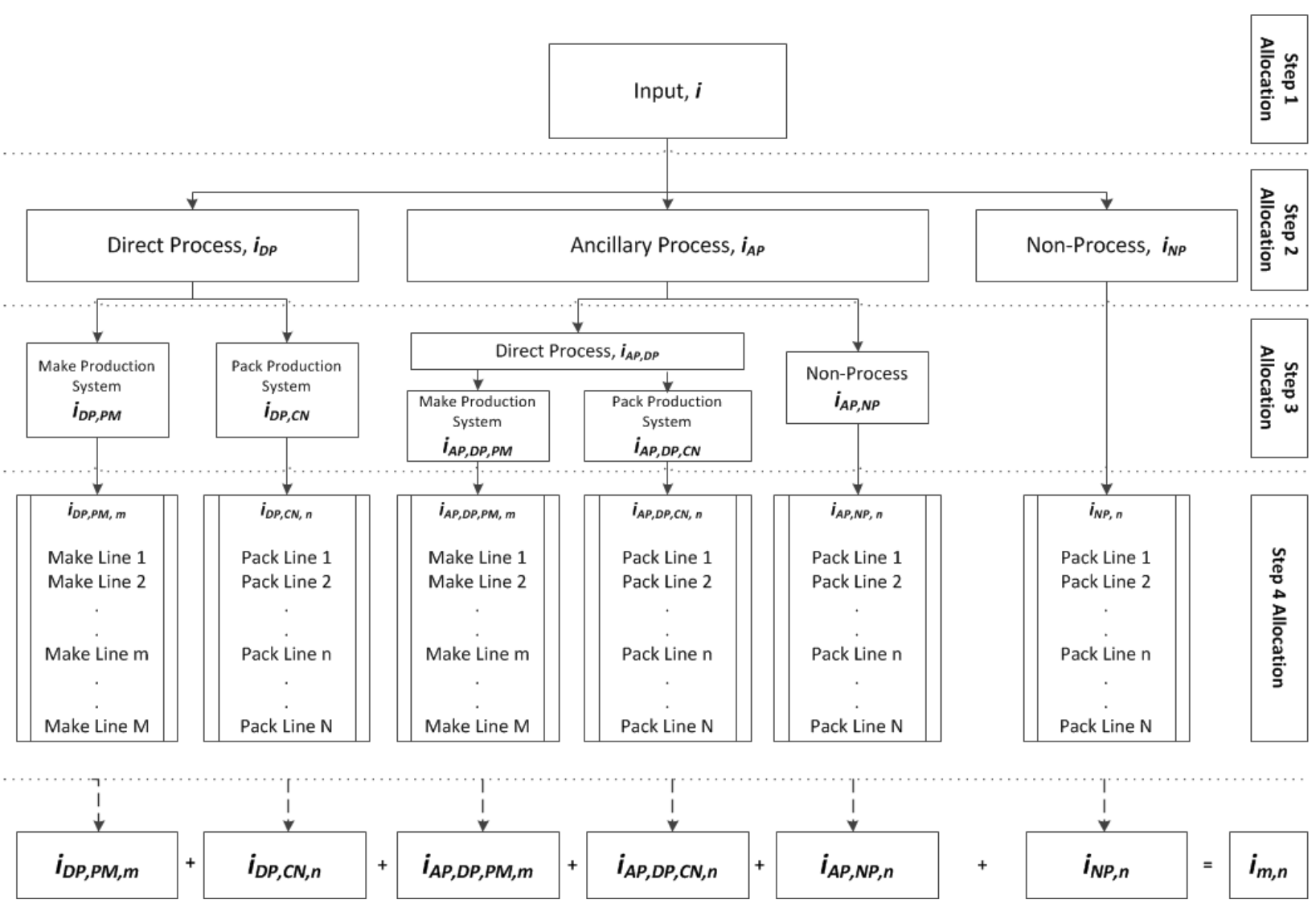

Figure 4. Multiscale allocation scheme for inputs of materials, utilities and labor

$i=i_{D P}+i_{A P}+i_{N P}$

and,

$i_{D P}=f^{i}{ }_{D P} \times i$

$i_{A P}=f^{i}{ }_{A P} \times i$

$i_{N P}=f^{i}{ }_{N P} \times i$

$f^{i}{ }_{D P}+f^{i}{ }_{A P}+f^{i}{ }_{N P}=1$ 

on the fraction $f$ denotes that the fraction is specific to a given input $i$ (for outputs an $o$ superscript is used). For an industrial process, these fractions are known parameters or can be calculated. For example, a chemical used in the production of intermediate will solely be used in the DP, and $f^{i}{ }_{D P}=1$. In some special cases where the input is shared among DP, AP and NP, such as electricity, the $f^{i}{ }_{D P}, f^{i}{ }_{A P}$ and $f^{i}{ }_{N P}$ fractions are calculated in a Step 0.

\subsubsection{Step 3 Allocation:}

In the Step 3 allocation, expansion of the Direct Process is required to represent all applicable production systems. In this case study, the Direct Process is subdivided into Make Lines and Pack Lines. $P M$ is used to denote $M$ paper making lines, and $C N$ denotes $N$ converting lines. Also, the inputs to the Ancillary Process are made suitable for use in Direct Process and Non-Process. For example, for boilers, natural gas is converted to steam and heat, and for water treatment, municipal water is transformed to treated water for process use. Step 3 makes use of Step 0 calculations which representatively split these inputs between the DP and NP through utilization in the AP.

\section{Distribution of Direct Process Inputs to Make Production System and Pack Production System}

The Direct Process inputs are composed of paper making and converting inputs,

$$
\begin{gathered}
i_{D P}=i_{D P, P M}+i_{D P, C N} \\
i_{D P, P M}=f^{i}{ }_{D P, P M} \times i_{D P} \\
(7) \\
i_{D P, C N}=f^{i}{ }_{D P, C N} \times i_{D P} \\
f^{i}{ }_{D P, P M}+f^{i}{ }_{D P, C N}=1
\end{gathered}
$$

Distribution of Ancillary Process Inputs to Direct Process (Make Production System and Pack Production System) and Non-Process

Equations for Ancillary Process inputs are somewhat similar to the Direct Process inputs; however, Ancillary Process inputs are split into Direct and Non-Process inputs. In addition, the inputs from Ancillary Process to Direct Process are split between the make and pack production systems.

$$
\begin{aligned}
& i_{A P}=i_{A P, D P}+i_{A P, N P}=\left(i_{A P, D P, P M}+i_{A P, D P, C N}\right)+i_{A P, N P} \\
& i_{A P, D P, P M}=f^{i}{ }_{A P, D P, P M} \times i_{A P} \\
& i_{A P, D P, C N}=f^{i}{ }_{A P, D P, C N} \times i_{A P}
\end{aligned}
$$




$$
\begin{aligned}
& i_{A P, N P}=f^{i}{ }_{A P, N P} \times i_{A P} \\
& f^{i}{ }_{A P, D P, P M}+f^{i}{ }_{A P, D P, C N}+f^{i}{ }_{A P, N P}=1
\end{aligned}
$$

\subsubsection{Step 4 Allocation:}

In the Step 4 allocation, the production systems are further subdivided into the production lines. In Equations 15 and 16, the inputs to the make production system and pack production system are expressed as a sum of inputs to the $m$ and $n$ production lines. Equations 17 and 18 are expressed as a summation of the inputs to the ancillary process that are used by the $m$ and $n$ production lines. In this work it is assumed that the inputs to the Non-Processes are distributed among the production lines (in this case, the pack production lines) which manufacture the final product from the facility. In Equation 19, the inputs to the Ancillary Process that are used by Non-Process are allocated to the pack production lines. Equation 20 assumes that the inputs to the Non-Process are distributed among the pack production lines.

$$
\begin{aligned}
& i_{D P, P M}=\sum_{M} i_{D P, P M, m} \\
& i_{D P, C N}=\sum_{N} i_{D P, C N, n} \\
& i_{A P, D P, P M}=\sum_{M} i_{A P, D P, P M, m} \\
& i_{A P, D P, C N}=\sum_{N} i_{A P, D P, C N, n} \\
& i_{A P, N P}=\sum_{N} i_{A P, N P, n} \\
& i_{N P}=\sum_{N} i_{N P, n}
\end{aligned}
$$

Thus, at the end of Step 4 allocation, we have subdivided the facility level inputs to obtain the distribution in the production line level inputs as shown in Equation 21.

$$
\begin{aligned}
& \underset{\text { Step } 1}{i}=\underbrace{i_{D P}+i_{A P}+i_{N P}}_{\text {Step } 2}=\underbrace{\left(i_{D P, P M}+i_{D P, C N}\right)+\left(i_{A P, D P, P M}+i_{A P, D P, C N}+i_{A P, N P}\right)+i_{N P}}_{\text {Step } 3} \\
& =\underbrace{\left(\sum_{M} i_{D P, P M, m}+\sum_{N} i_{D P, C N, n}\right)+\left(\sum_{M} i_{A P, D P, P M, m}+\sum_{N} i_{A P, D P, C N, n}+\sum_{N} i_{A P, N P, n}\right)+\sum_{N} i_{N P, n}}_{\text {Step } 4}
\end{aligned}
$$




\subsubsection{Step 0 for Intermediate and Product Distribution among Production Lines:}

Multiple Step 0 calculations are required to compute the terms in Equation 21. In this contribution an example of the Step 0 calculation for the intermediate and product distribution among the production systems is shown in Table 3. The lowest level of granularity in this problem is the production line, i.e. the make line or pack line. Each of these production lines have a primary output, the Intermediate $I$ for Make Lines and the Product $P$ for the Pack Lines. Table 3 provides a distribution of the inputs, outputs, intermediates, and products and expresses the fractions for a particular Production Line.

Table 3. Step 0 fractions used in calculation for intermediate and product distribution among production systems

\begin{tabular}{|l|l|l|}
\hline Fraction Type & Make/Pack Line & Expression \\
\hline Input $i$ & To Make Line $m$ & $\frac{i_{D P, P M, m}}{\sum_{M} i_{D P, P M, m}}$ \\
\hline Input $i$ & To Pack Line $n$ & $\frac{i_{D P, C N, n}}{\sum_{N} i_{D P, C N, n}}$ \\
\hline Output $o$ & & $\frac{o_{D P, P M, m}}{\sum_{M} o_{D P, P M, m}}$ \\
\hline Output $o$ & From Make Line $m$ & $\frac{o_{D P, C N, n}}{\sum_{N} o_{D P, C N, n}}$ \\
\hline Intermediate $I$ & From Pack Line $n$ & $\frac{I_{m}}{\sum_{M} I_{m}}$ \\
\hline Product $P$ & Produced in Make Line $m$ & $\frac{P_{n}}{\sum_{N} P_{n}}$ \\
\hline & Produced in Pack Line $n$ & \\
\hline
\end{tabular}

Determining these fractions can be done using one of the proposed allocation approaches in the ISO 14044 standard, including using a physical unit, such as mass or energy, or an economic unit, such as cost. In this case study, the facility produces a variety of tissue and towel products. Since the studied production system involves primarily a physical transformation and the majority of data available are mass-based, mass was used for allocation. However, future analyses employing energy and market-value based allocation as well as unit operation level granularity data (non-allocation) would be useful to determine advantages, applicability, and weaknesses from application of different allocation approaches.

\subsubsection{Calculation of $i_{m, n}$ :}


The terms in $i_{m, n}$ as shown in Figure 4 can be computed using the scaling method explained in Figure 3 and Table 2. Equations 22 and 23 give the values according to the two options of $I_{m} / I_{n}$.

When $I_{m} / I_{n}<1$, $i_{m, n}=\left(i_{D P, P M, m}+i_{D P, C N, n} \times I_{m} / I_{n}\right)+\left(i_{A P, D P, P M, m}+i_{A P, D P, C N, n} \times I_{m} / I_{n}+i_{A P, N P, n} \times I_{m} / I_{n}\right)+i_{N P, n} \times I_{m} / I_{n}$

When $I_{m} / I_{n}>1$, $i_{m, n}=\left(i_{D P, P M, m} \times I_{n} / I_{m}+i_{D P, C N, n}\right)+\left(i_{A P, D P, P M, m} \times I_{n} / I_{m}+i_{A P, D P, C N, n}+i_{A P, N P, n}\right)+i_{N P, n}$

As discussed earlier, $I_{m} / I_{n}$ is a known value determined from process data. The unknown terms in Equations 22 and 23 are expressed as given in Equations 24-29, which will be explained by example of Equation 24. $i_{D P, P M, m}$ is an input to the Direct Process, make production system, and $m^{\text {th }}$ make line. The numerator and denominator of Equation 24 are multiplied by $\sum_{M} i_{D P, P M, m}$, the sum of inputs to the Make Lines, i.e., the total input to the Make Production System. $\frac{i_{D P, P M, m}}{\sum_{M} i_{D P, P M, m}}$ gives the fraction of inputs to the $m^{\text {th }}$ Make Line and ideally, is a known quantity from the Step 0 calculation as explained above. However, when this quantity is not known or proprietary rights prevent a company from disclosing such information, the term may be approximated according to the fraction of intermediate or final product from the Production Line. Thus, a Step 0 calculation of only the fraction of intermediate or product from a particular Production Line will be sufficient to estimate the fraction of input it requires. In this case, we have approximated $\frac{i_{D P, P M, m}}{\sum_{M} i_{D P, P M, m}} \approx \frac{I_{m}}{\sum_{M} I_{m}}$, i.e., the fraction of inputs to the $m^{\text {th }}$ Make Line is similar to the fraction of intermediate produced from the same line. Similarly, in Equation 25, the fraction of inputs to the $n^{\text {th }}$ Pack Line is assumed equivalent to the fraction of final product manufactured from the line. Using similar approximations, Equations 26-29 are computed. It should be noted here that to obtain improved IPSA output values the actual fraction of inputs to the production line of interest should always be chosen when available to obtain a better subdivision of the process inputs.

$$
\begin{aligned}
& i_{D P, P M, m}=\frac{i_{D P, P M, m}}{\sum_{M} i_{D P, P M, m}} \times \sum_{M} i_{D P, P M, m} \approx \frac{I_{m}}{\sum_{M} I_{m}} \times \sum_{M} i_{D P, P M, m}=\frac{I_{m}}{\sum_{M} I_{m}} \times i_{D P, P M} \\
& i_{D P, C N, n}=\frac{i_{D P, C N, n}}{\sum_{N} i_{D P, C N, n}} \times \sum_{N} i_{D P, C N, n} \approx \frac{P_{n}}{\sum_{N} P_{n}} \times \sum_{N} i_{D P, C N, n}=\frac{P_{n}}{\sum_{N} P_{n}} \times i_{D P, C N}
\end{aligned}
$$


Thus, all the terms in Equations 22 and 23 are computed using Equations 24-29. Now the aim is to substitute each component in Equations 22 and 23 to obtain the values in terms of input $i$. The following equations give the values for the different components for $i_{m, n}$.

Substituting equations from Step 2 and Step 3 allocation in Equations 24-29, one gets

$$
\begin{aligned}
& i_{D P, P M, m}=\frac{I_{m}}{\sum_{M} I_{m}} \times f^{i}{ }_{D P, P M} \times f^{i}{ }_{D P} \times i \\
& i_{D P, C N, n}=\frac{P_{n}}{\sum_{N} P_{n}} \times f^{i}{ }_{D P, C N} \times f^{i}{ }_{D P} \times i \\
& i_{A P, D P, P M, m}=\frac{I_{m}}{\sum_{M} I_{m}} \times f^{i}{ }_{A P, D P, P M} \times f^{i}{ }_{A P} \times i \\
& i_{A P, D P, C N, n}=\frac{P_{n}}{\sum_{N} P_{n}} \times f^{i}{ }_{A P, D P, C N} \times f^{i}{ }_{A P} \times i \\
& i_{A P, N P, n}= \\
& \sum_{N} P_{n} \times f_{A P, N P}^{i} \times f^{i}{ }_{A P} \times i
\end{aligned}
$$




$$
i_{N P, n}=\frac{P_{n}}{\sum_{N} P_{n}} \times f^{i}{ }_{N P} \times i
$$

Finally, substituting Equations 30-35 in Equation 22, when $I_{m} / I_{n}<1$, one gets,

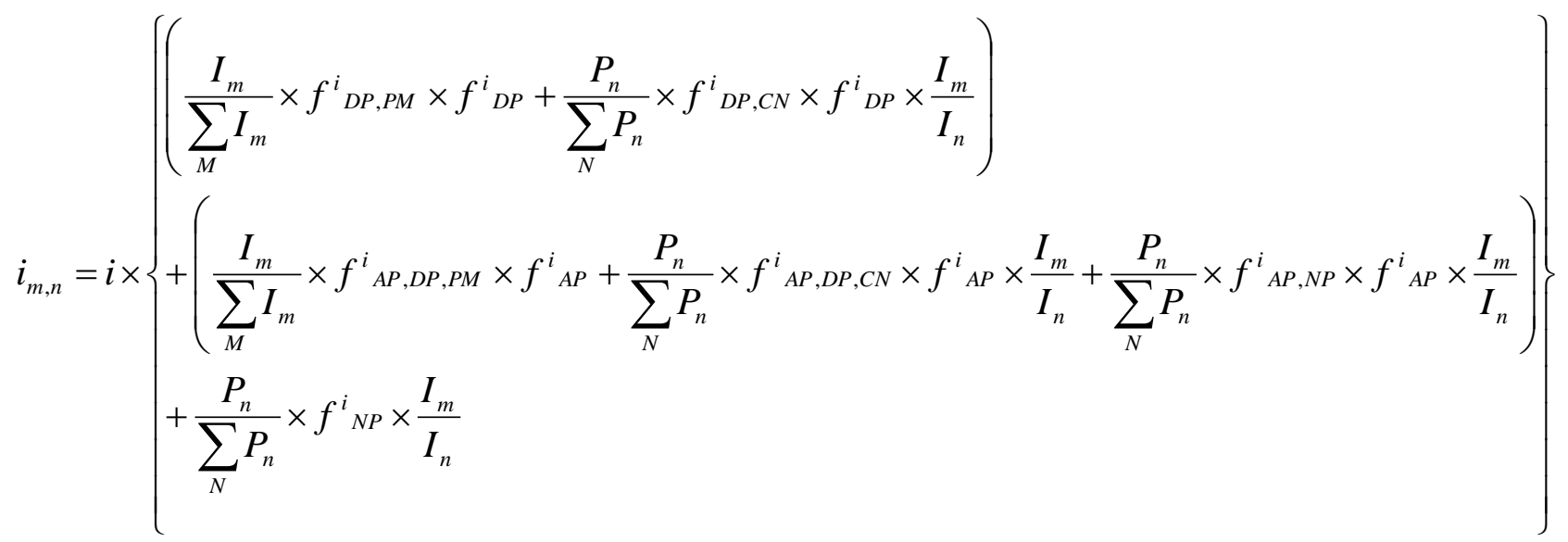

And substituting Equations 30-35 in Equation 23, when $I_{m} / I_{n}>1$, we get,

$$
\begin{aligned}
& \int\left(\frac{I_{m}}{\sum_{M} I_{m}} \times f^{i}{ }_{D P, P M} \times f^{i}{ }_{D P} \times \frac{I_{n}}{I_{m}}+\frac{P_{n}}{\sum_{N} P_{n}} \times f^{i}{ }_{D P, C N} \times f^{i}{ }_{D P}\right) \\
& \left.i_{m, n}=i \times\left\{\begin{array}{l}
+\left(\frac{I_{m}}{\sum_{M} I_{m}} \times f^{i}{ }_{A P, D P, P M} \times f^{i}{ }_{A P} \times \frac{I_{n}}{I_{m}}+\frac{P_{n}}{\sum_{N} P_{n}} \times f^{i}{ }_{A P, D P, C N} \times f^{i}{ }_{A P}+\frac{P_{n}}{\sum_{N} P_{n}} \times f^{i}{ }_{A P, N P} \times f^{i}{ }_{A P}\right.
\end{array}\right\}\right)
\end{aligned}
$$




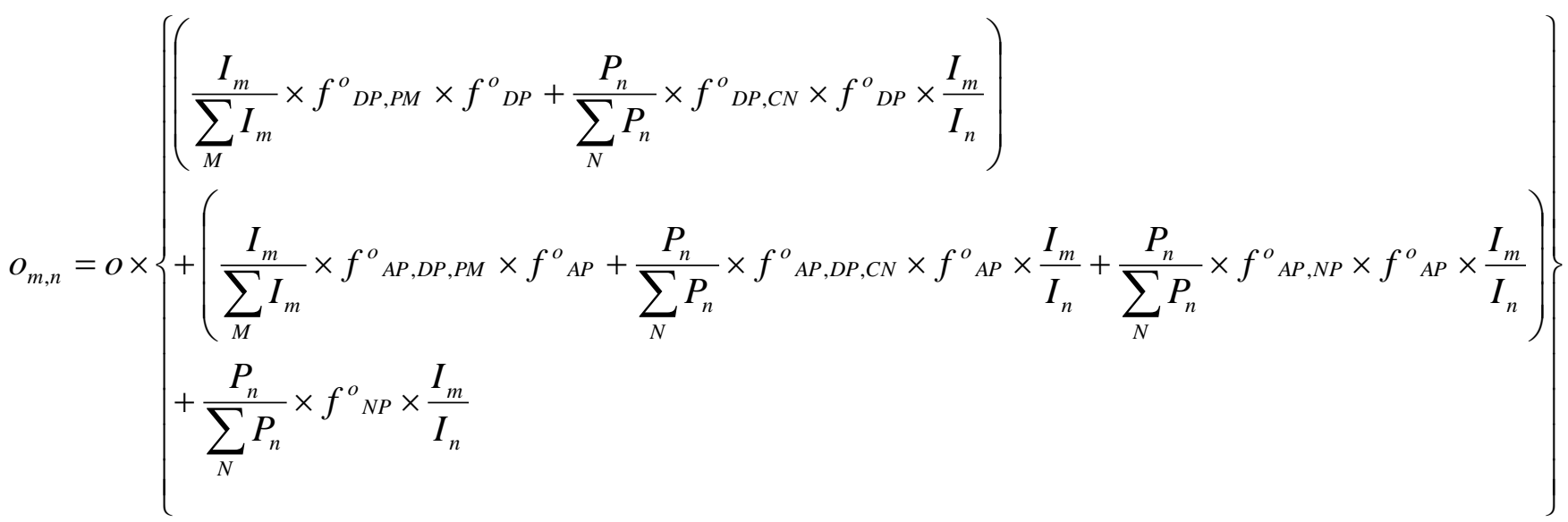

And when $I_{m} / I_{n}>1$,

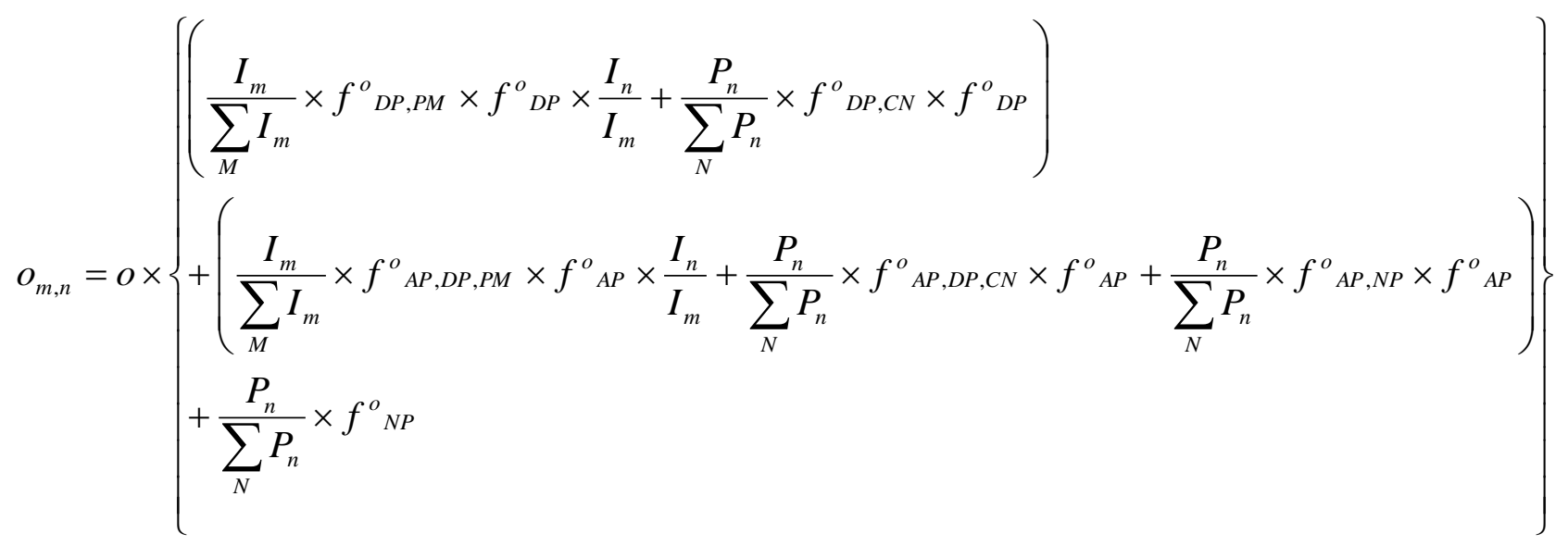

\subsubsection{Calculation of Inputs and Outputs for Unit Quantity of Product:}

Equations 36-39 give the inputs and outputs related to the manufacturing of the final product from a combination of production lines of interest. The final value of inputs and outputs per unit of production is obtained using the following relation.

$$
\begin{gathered}
\hat{i}_{m, n}=\frac{i_{m, n}}{P_{n}} \\
\hat{o}_{m, n}=\frac{o_{m, n}}{P_{n}}
\end{gathered}
$$




\subsubsection{Comparing Results to Aggregate Allocation Approach (facility-level allocation vs. subprocess level allocation):}

For a typical LCA, the inputs and outputs to the facility are grossly allocated using the final product distribution from the facility in an aggregate approach. Such methods have been shown to generate different results than those obtained with a subprocess level allocation (Wang et al., 2004). In this paper, the facility level allocation is obtained from Equations 42 and 43 . These results can be compared with the allocation obtained from Equations 36-41 to determine the difference in the inputs and outputs allocated to the product of interest.

$$
\begin{aligned}
& i_{m, n}^{\prime}=i \times \frac{P_{n}}{\sum_{N} P_{n}} ; \hat{i}_{m, n}^{\prime}=\frac{i}{\sum_{N} P_{n}} \\
& o_{m, n}^{\prime}=o \times \frac{P_{n}}{\sum_{N} P_{n}} ; \quad \hat{o}_{m, n}^{\prime}=\frac{o}{\sum_{N} P_{n}}
\end{aligned}
$$

As such, the aggregate allocation approach inappropriately creates a bias towards average facility values for the inputs and outputs allocated to the product of interest. The IPSA method provides an advantage by subdividing the process according to multiscale model decomposition and helps to reduce the error in accounting for the inputs and outputs related to a product. Additionally, IPSA can potentially identify and quantify losses, which can be examined to reveal process inefficiencies or errors in the step allocation of facility inputs and output values.

\section{Conclusions}

The Industrial Process System Assessment method has been described herein to assist with the assignment of allocation to the multitude of inputs and outputs across the Direct Processes, Non-Processes, and Ancillary Processes for a complex manufacturing facility. This is a significant contribution in establishing a representative and accurate life cycle inventory for a manufacturing process under a sustainability evaluation. This method will enable more accurate estimation of environmental impact for products from complex multi-line and multi-product facilities than is provided by the allocation procedures commonly used in LCA.

A four step multiscale model of an industrial process system assessment is developed in this paper. This model is then used to formulate the equations required to connect facility level inputs and outputs to the products of interest. In this method, the information exchange among three different scales of IPSA has been demonstrated. Besides serving the purpose of allocation in a gate-to-gate LCA for a desired product, the IPSA method provides a potential method for loss accounting within a process system. 
The ability to have a greater quantity and higher accuracy of data at the point of use will not only strengthen the proper allocation contributions from that point of use, but also the data can be informative in determining scheduled and unscheduled events (e.g., risks, fault), opportunities for control, optimization, and process design improvements, and uses for optimal placement of sensors and meters (e.g., product quality).

By having more granular information about a process, such as that provided by the IPSA method, there are opportunities for a more thorough comprehension of the process, which can lead to improved sustainability of the supply chain. A future addition of the unit operation level granularity in the IPSA model will allow for a greater understanding in the relationship between facility inputs and outputs. This is anticipated to allow for improved allocation based on physical and chemical phenomena within the process system and provide an improved facility model that can assist with the design and evaluation of complex industrial production systems. Thus, the IPSA method provides a robust mathematical model of an industrial process system to incorporate subprocess level information, which can be used for allocation of inputs and outputs from multiple product systems.

\section{Disclaimer}

This article does not reflect the endorsement or opinion of the U.S. Environmental Protection Agency or P\&G.

\section{Acknowledgement}

This project was supported in part by Dr. Sengupta's appointment to the Research Participation Program at the Office of Research and Development, National Risk Management Research Laboratory, U.S. Environmental Protection Agency, administered by the Oak Ridge Institute for Science and Education through an interagency agreement between the U.S. Department of Energy and EPA. A collaboration research and development agreement between the US EPA and Procter \& Gamble on Sustainable Supply Chain Design enabled this research project.

\section{Acronyms}

IPSA: Industrial Process System Assessment

LCA: Life Cycle Assessment

LCI: Life Cycle Inventory 


\section{References}

Azapagic, A., Clift, R., 1999a. Allocation of environmental burdens in co-product systems: Product-related burdens (Part 1). Int J LCA 4, 357-369.

Azapagic, A., Clift, R., 1999b. Allocation of environmental burdens in multiple-function systems. J. Clean. Prod. 7, 101-119.

Azapagic, A., Clift, R., 2000. Allocation of environmental burdens in co-product systems: Process and product-related burdens (part 2). Int J LCA 5, 31-36.

Burgess, A.A., Brennan, D.J., 2001. Application of life cycle assessment to chemical processes. Chem. Eng. Sci. 56, 2589-2604.

Cameron, I.T., Ingram, G.D., Hangos, K.M., 2008. Multiscale Process Modeling, in: Puigjaner,

L., Heyen, G. (Eds.), Computer Aided Process and Product Engineering. Wiley-VCH Verlag GmbH, pp. 189-221.

Curran, M.A., 2007. Studying the Effect on System Preference by Varying Coproduct Allocation in Creating Life-Cycle Inventory. Environ. Sci. Technol. 41, 7145-7151.

Dominguez-Ramos, A., Chavan, K., García, V., Jimeno, G., Albo, J., Marathe, K.V., Yadav, G.D., Irabien, A., 2014. Arsenic Removal from Natural Waters by Adsorption or Ion Exchange: An Environmental Sustainability Assessment. Ind. Eng. Chem. Res.

Ekvall, T., Finnveden, G., 2001. Allocation in ISO 14041-a critical review. J. Clean. Prod. 9, 197-208.

González-García, S., Castanheira, É.G., Dias, A.C., Arroja, L., 2013. Environmental performance of a Portuguese mature cheese-making dairy mill. J. Clean. Prod. 41, 65-73.

Grossmann, I.E., 2004. Challenges in the new millennium: product discovery and design, enterprise and supply chain optimization, global life cycle assessment. Comput. Chem. Eng. 29, 29-39.

Grossmann, I.E., Westerberg, A.W., 2000. Research challenges in process systems engineering. AICHE J. 46, 1700-1703.

Ingwersen, W., Cabezas, H., Weisbrod, A.V., Eason, T., Demeke, B., Ma, C., Lee, S.J., Hawkins, T., Bare, J.C., Ceja, M., 2014. Integrated Metrics for Improving the Life Cycle Approach to Assessing Product System Sustainability. Sustainability 6, 1386-1413.

ISO, 2006. 14044: environmental management-life cycle assessment-requirements and guidelines. International Standards Organization.

Jiménez-González, C., Curzons, A., Constable, D., Cunningham, V., 2004. Cradle-to-gate life cycle inventory and assessment of pharmaceutical compounds. Int J LCA 9, 114-121.

Jiménez-González, C., Kim, S., Overcash, M., 2000. Methodology for developing gate-to-gate Life cycle inventory information. Int J LCA 5, 153-159.

Jin, M., Li, Y., Tsung, F., 2010. Chart allocation strategy for serial-parallel multistage manufacturing processes. IIE Transactions 42, 577-588.

Lindfors, L.G., 1995. Nordic guidelines on life-cycle assessment. Nordic Council of Ministers.

Löfgren, B., Tillman, A.-M., 2011. Relating manufacturing system configuration to life-cycle environmental performance: discrete-event simulation supplemented with LCA. J. Clean. Prod. 19, 2015-2024.

Löfgren, B., Tillman, A.-M., Rinde, B., 2011. Manufacturing actor's LCA. J. Clean. Prod. 19, 2025-2033. 
Power, W., 2009. Life Cycle Management: How business uses it to decrease footprint, create opportunities and make value chains more sustainable. UNEP-SETAC Life Cycle Initiative, Paris.

Sammons Jr, N.E., Yuan, W., Eden, M.R., Aksoy, B., Cullinan, H.T., 2008. Optimal biorefinery product allocation by combining process and economic modeling. Chem. Eng. Res. Des. 86, 800-808.

Sonnemann, G., Vigon, B., 2011. Global Guidance Principles for Life Cycle Assessment Databases (Shonan Guidance Principles). UNEP-SETAC Life Cycle Initiative, Paris.

USEPA, 1993. Life-Cycle Assessment: Inventory Guidelines and Principles.

USEPA, 2006. Life Cycle Assessment: Principles and Practice.

Wan Alwi, S.R., Manan, Z.A., Klemeš, J.J., Huisingh, D., 2014. Sustainability engineering for the future. J. Clean. Prod. 71, 1-10.

Wang, M., Lee, H., Molburg, J., 2004. Allocation of energy use in petroleum refineries to petroleum products. Int. J. LCA 9, 34-44.

Weisbrod, A., Bare, J., Ceja, M., Ingwersen, W., Young, D., Smith, R., Demeke, B., Weber, B., Lee, S.-J., Gausman, M., Sengupta, D., Abraham, J., Hawkins, T., Zanoli, E., Alavarez, M., Ma, C., Eason, T., Cabezas, H., Gonzalez, M., Ruiz-Mercado, G., Armstrong, B., 2013. A Government and Industry Application of LCA and Novel Sustainability Metrics: Part 1, Society of Environmental Toxicology \& Chemistry North America (SETAC-NA) Annual Meeting, Nashville, TN.

\section{Figure Captions}

Figure 1. Multiple scales of process engineering (adapted from Cameron et al., 2008; shaded area includes the scales modeled in the IPSA method.

Figure 2 (a) Step 1: Facility Flows, (b) Step 2: Process Classification, (c) Step 3: Production System Definition, and (d) Step 4: Allocation at Multiple Scales.

Figure 3. Scaling method for computing the intermediate ratio between the "Make Line $m$ " and the "Pack Line $n$ "

Figure 4. Multiscale allocation scheme for inputs of materials, utilities and labor

Figure 5. Multiscale allocation scheme for outputs of products and environmental releases 